\title{
Marine Radar Derived Current Vector Mapping at a Planned Commercial Tidal Stream Turbine Array in the Pentland Firth, U.K.
}

\author{
David L. McCann and Paul S. Bell \\ National Oceanography Centre \\ Liverpool, U.K. \\ dmca@noc.ac.uk
}

\begin{abstract}
A marine radar was deployed on a remote clifftop overlooking a $4.8 \mathrm{~km}$ radius area of the Inner Sound of Stroma in the Pentland Firth for 3 months during spring 2013. The area viewed by the radar includes The Crown Estate lease areas for MeyGen Ltd (Inner Sound of Stroma) and Scottish Power Renewables (Ness of Duncansby), although the data analysis has focussed solely on the MeyGen area. Data were post processed to extract current vector maps based on determining the Doppler shift of sea surface waves by the tidal current. Comparisons between current time series from two Acoustic Doppler Current Profiler (ADCP) surveys and the radar derived data are presented and show excellent correlation. The quality of the data has enabled tidal analyses to be performed and spatial variations in tidal current constituents to be mapped.
\end{abstract}

Keywords-Marine renewables; Radar; Tidal current mapping

\section{INTRODUCTION}

MeyGen Ltd. is a marine renewable energy (MRE) company currently planning to install one of the world's first tidal stream turbine arrays in the tidal race of the Inner Sound of Stroma, North Scotland (Fig. 1). MeyGen have recently been granted consent by the Scottish government to develop an 86MW primary stage installation (Phase 1) with an initial 6MW demonstration array (Phase 1a) of 4 turbines, the first turbine having a planned deployment date in 2015. MeyGen is also in competition for the Scottish Government's $£ 10 \mathrm{M}$ 'Saltire prize', the winner of which will be the individual, company or organisation that produces the most amount of marine renewable energy over a two year period with a baseline of 100GWh by January 2017.

Following the deployment of a similar radar system at the European Marine Energy Centre (EMEC) as part of the FLOWBEC project [1], in 2013 the National Oceanography Centre (NOC), U.K., undertook a Natural Environment Research Council (NERC) funded Marine Renewables Knowledge Exchange Program (MRE-KEP) internship with MeyGen Ltd. The purpose of the KE internship was to showcase the state-of-the-art in the remote sensing of tidal currents with X-band marine radar and to help MeyGen gain valuable knowledge of the waters of the Inner Sound.

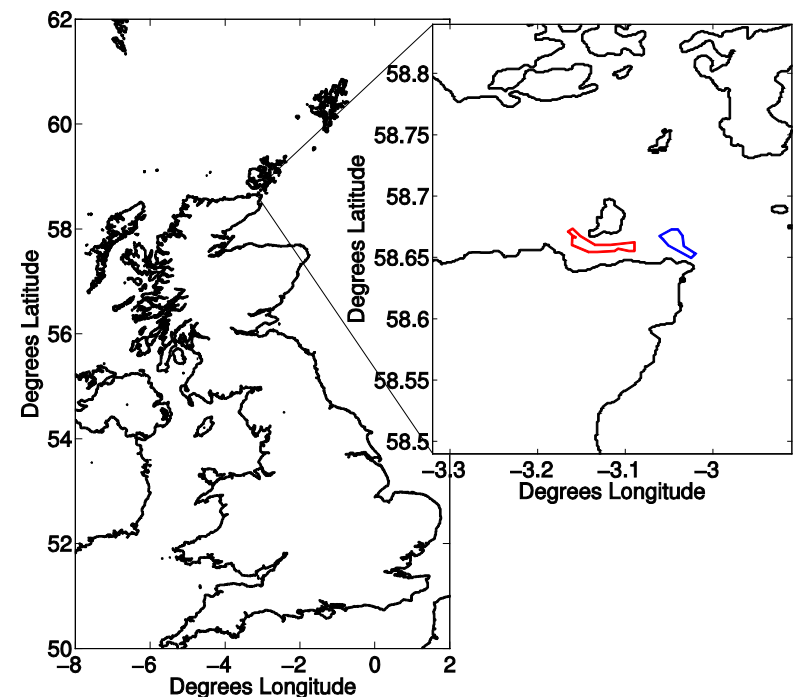

Fig. 1. Location of the project area and the extent of the MeyGen Inner Sound of Stroma (red, inset) and the nearby Scottish Power Renewables Ness of Duncansby (blue, inset) lease areas.

At present, measurements of tidal currents involve high cost, in-situ deployments of current meters, usually at a low number of specific locations, which involve a significant risk of equipment loss or damage in such high energy environments. Such data can then be used to calibrate numerical models from which a more detailed understanding of the spatial variability may be derived to aid decision making on turbine positions and facilitate predictions of energy yield. The ability to collect time series of current vector maps using a shore based system will add value to insitu measurements by enabling validation of model outputs at a large number of points across a site of interest and placing the in-situ measurements into a broader context, reducing risk and increasing confidence in resource assessments and turbine placement decisions. 


\section{Methodology}

The radar installation consisted of a Kelvin Hughes $10 \mathrm{~kW}$ X-band unit with a $2.4 \mathrm{~m}$, horizontally-polarised antenna mounted on a scaffold approximately $12 \mathrm{~m}$ above mean sea level with a rotation rate of $\sim 24 \mathrm{rpm}$ (Fig. 2). The radar transceiver and processing computer were housed in an enclosure built into the scaffold for stability. Due to the remote nature of the field site power was supplied by a battery bank (supplied by OffGrid Energy ltd.), charged periodically and automatically by a $6 \mathrm{kVA}$ diesel generator with remote start capabilities. The peak power requirement of the entire system was very low at approximately 360VA.

The raw radar video signal was intercepted by a WaMoS II radar computer (OceanWaveS $\mathrm{GmbH}$ ) and converted into time-stamped, digitised images of radar backscatter intensity, an example of which can be seen in Fig. 3. The installation was in constant operation between $5^{\text {th }}$ March and $11^{\text {th }}$ June 2013 and was set to record for intervals of 128 images ( 5.5 minutes) every 20 minutes.

Due to the requirements of the project and the need for analytical expediency, the analysis area was restricted to cover the MeyGen lease site (white and red lines respectively, Fig. 3 ). The NOC current analysis method involves the extraction of spectral wave parameters from time-indexed radar images of waves on the sea surface - visible due to the phenomenon of Bragg resonant scattering of incident microwaves (wave patterns, or 'sea clutter' on Fig. 3). The wave parameters are then used to resolve the effects on the wave dispersion of an unknown depth and unknown Doppler shift by the mean current; a process known as inversion. Further information on the wave inversion method can be found in [2], [3], [4] and [5]. The calculation of wave parameters requires the analysis of a finite area of sea surface carrying the assumption that the wavenumber and frequency spectra across the discrete analysis area are homogenous.

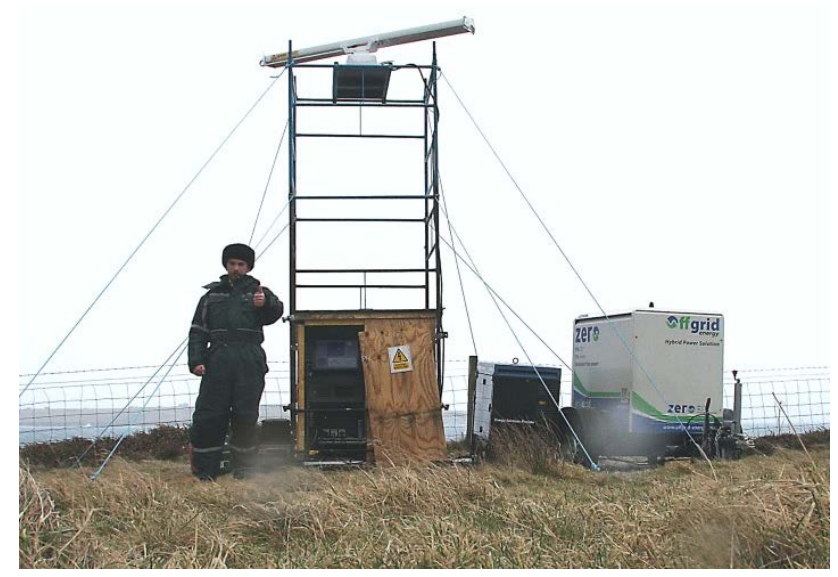

Fig 2. Radar field deployment on a cliff-top overlooking the Inner Sound with NOC scientist for scale.



Fig. 3. Example of a single radar image, showing the signals from large, Easterly waves (sea-clutter).

The analysis algorithm therefore works on areas of sea surface typically $400-800 \mathrm{~m}$ across and transposes this area step-wise at a quarter of that spacing (200m in this case) in order to produce a map of calculated velocity component magnitudes. Intensive quality control (QC) techniques developed at the NOC are employed on the calculated currents to minimise the secondary effect of poorly resolved current vectors on subsequent analyses. This is vitally important as poor wave data (a product of low wind / waves) and meteorological effects can have a significant impact on the quality of calculated currents and any subsequent analyses of those currents.

Calculated mean flow speeds were validated against two in-situ $600 \mathrm{kHz}$ 4-beam Acoustic Doppler Current Profiler (ADCP) deployments that overlapped portions of the radar survey period. The Environmental Research Institute (ERI) survey was located at the cross symbol on Fig. 3 and covered 14 days of the data set. The MeyGen (MG) survey was located at the plus symbol on Fig. 3 and covered 24 days of the data set. There is yet an undetermined relationship between the current derived from X-band radar and the current the waves actually 'feel'. However the current hypothesis is that the radar derived current corresponds approximately to the depthmean flow speed in these water depths $(\sim 30 \mathrm{~m})$. The depthaveraged ADCP velocities were therefore used in the validation.

Tidal harmonic analysis was performed on the radar derived $u$ and $v$ horizontal current vectors, employing the open-source tidal analysis and prediction toolbox 'UTide' [6] which was chosen due to its ability to handle non-continuous data series. UTide attempts to fit up to $40+$ tidal constituents to a tidal current record, discarding fits that show a poor signal to noise ratio. Harmonic analysis is very sensitive to the quality and record length of input data; both of which must be taken into consideration when interpreting the results.

\section{OBSERVATIONS}

Fig. 4 shows an example snapshot of tidal flow vectors derived from the NOC current analysis during a flood tide 




Fig. 4. Example tidal current vectors ( $\mathrm{ms}^{-1}$ ) computed from a record of radar images during a flood tide over the MeyGen lease area (black polygon). Grid points excluded by the QC routines are shown in grey.

across the MeyGen lease area. Over 7,500 of such records were present in the final data set, however due to the effect of QC (grey areas in Fig. 4.) the final number of good quality, reliable records in the set varied from $\sim 80 \%$ to $\sim 20 \%$ of those initially recorded.

Fig. 5 shows the results of the ADCP validation for the ERI dataset and Fig. 6 the results of the validation for the MG dataset. The $u$ component of the radar-derived current is shown to have an excellent correlation with the depthaveraged ADCP $u$-component velocities with $\mathrm{R}^{2}$ values of 0.98 for both the ERI and MG datasets. The $v$ component velocities are shown to have a very poor correlation-this is thought to be due to the comparison between a point (ADCP) and an area (radar) measurement. At the location of the ADCP surveys there are extensive sub-grid-scale cross-flow features and strong residual circulations originating from the inertial flow around the Isle of Stroma which cannot be resolved in the radar analysis due to its assumption of homogeneity across a grid cell (800m square). Waves are only Doppler shifted by currents with a component on the axis of wave propagation. This generally leads to a good determination of the current component in line with the wave propagation axis, but less confidence in the current component normal to the wave propagation axis. In reality, there is usually sufficient wave direction spread to allow a reasonable estimate of both current components, but the cross-wave component can be difficult to resolve in areas where there is very low wave directional spread. In this case, the location of the ADCP deployments in close proximity to the island of Stroma means that the waves are almost always directly in line with the tidal currents, and there is often very little cross-current wave component to assist in its determination.

It is expected that a comparison with an in-situ survey in a more open part of the study area exposed to a wider range of wave directions (e.g. towards the East of the MeyGen lease area, out of the influence of Stroma and the Inner Sound) would provide a much better validation for the cross-flow velocity component.

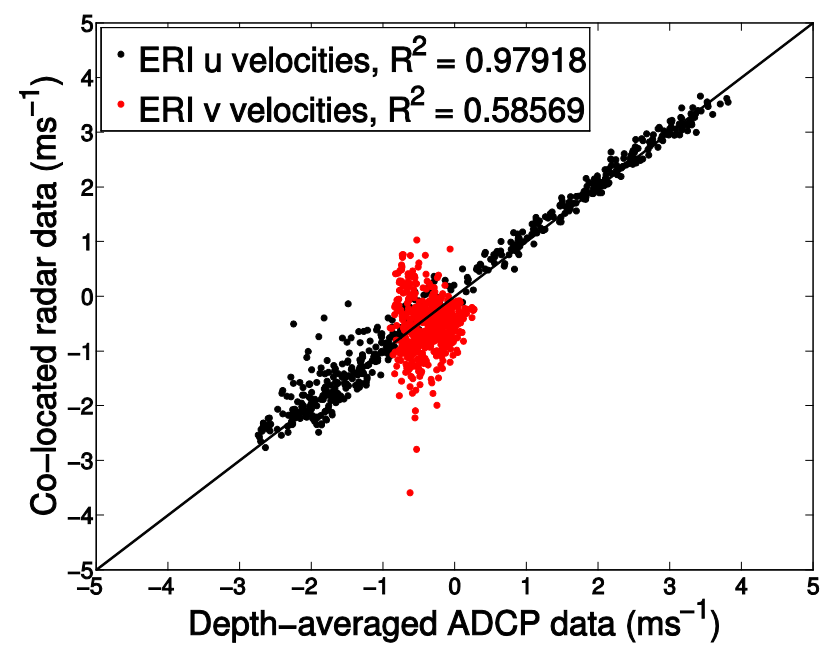

Fig. 5. Comparison between the depth-averaged ERI ADCP velocities $\left(\mathrm{ms}^{-1}\right)$ and co-located radar derived currents.

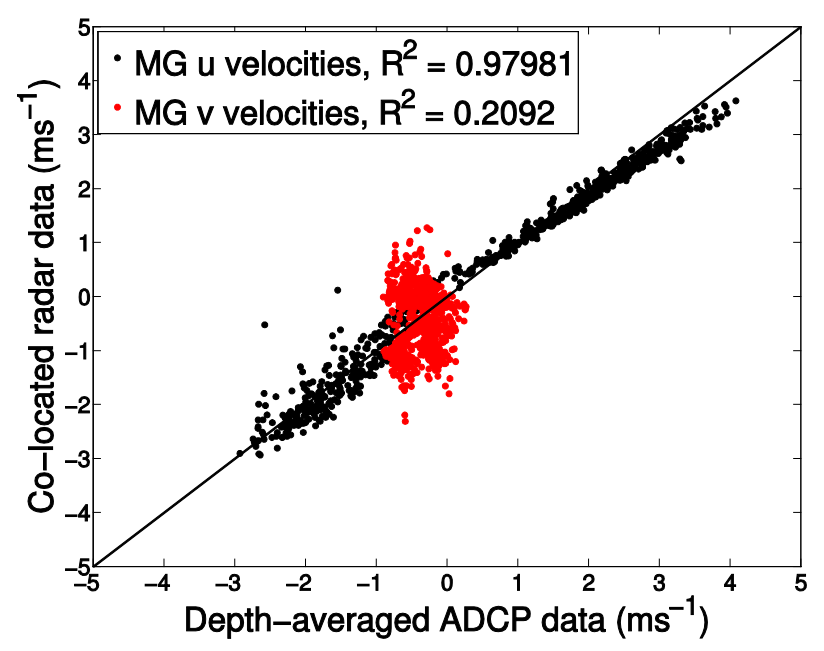

Fig. 6. Comparison between the depth-averaged MG ADCP velocities $\left(\mathrm{ms}^{-1}\right)$ and co-located radar derived currents. 
Harmonic analysis was applied to each grid point in the NOC radar derived dataset where sufficient data was available. Over 40 tidal harmonic constituents were fitted to the data set but the dominant harmonics were found to be restricted to the lunar and solar diurnal and semidiurnal with the majority of the spectral energy $(>80 \%)$ found in the principal lunar semidiurnal $\mathrm{M}_{2}$. Fig. 7 shows the absolute magnitudes of the amplitudes of the $\mathbf{M}_{2}$ tidal current constituent and Fig. 8 its phase relative to Greenwich.

The sensitivity of the analysis to poor data is visible in the noisy areas towards the north of the analysis area where wave shadowing by the island of Stroma and the prevalence of subgrid-scale flow features hinder the visibility of sea clutter on the raw data and thus the accurate resolution of flow vectors.

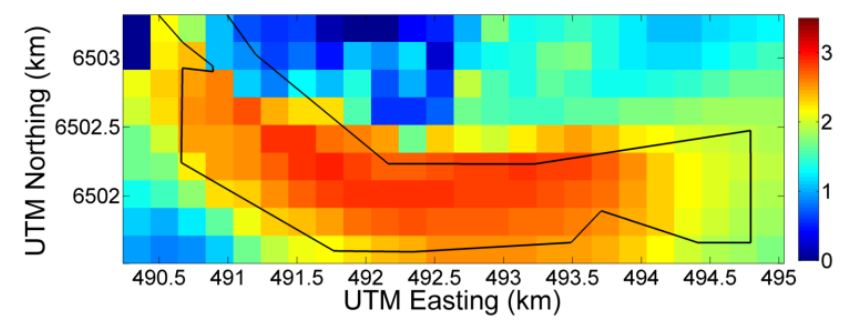

Fig. 7. The absolute magnitude of the $\mathrm{M}_{2}$ tidal constituent ( $\mathrm{ms}^{-1}$ ) from harmonic analysis of radar-derived currents.

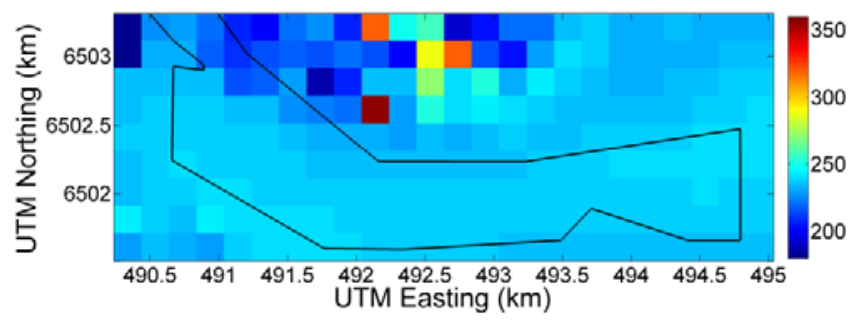

Fig. 7. The phase of the $\mathrm{M}_{2}$ tidal constituent (degrees relative to Greenwich) from harmonic analysis of radar-derived currents.

\section{CONCLUSIONS}

The application of X-band radar to an area as dynamic as the Inner Sound of Stroma was challenging both operationally and scientifically. However the resulting data set, although (at present) only covering the MeyGen lease area, has proven to be an extensive and useful addition to the knowledge of the hydrodynamics of the Inner Sound of Stroma.

Calculating the speed of tidal flow from a remote position on land using microwave images of the sea surface is a nontrivial exercise. With careful consideration of the limits of Xband radar images and the analytical methods applied to them, the resulting tidal current dataset is excellent, attested by the quality of the ADCP validation. It is therefore concluded that the radar-derived tidal current dataset from the NOC researchgrade analysis, once appropriate QC has been applied and limitations are taken into account, can be used for further model validation \& comparison and to form the basis of resource estimation and future locations of in-situ surveys.

\section{ACKNOWLEDGMENT}

This work was undertaken as part of a Knowledge Exchange internship funded by the Natural Environment Research Council through the National Oceanography Centre, grant reference NE/K50144X/1. The authors would like to thank Sarah Crammond at MeyGen and Philip Archer at Atlantis Resources Corporation together with Jason McIlvenny and Juvenal Dufaur for the provision of the two ADCP records. We would also like to thank Mr Alistair Cormack for the provision of the radar site and logistical help installing and maintaining the radar system.

\section{REFERENCES}

[1] P. S. Bell, D. L. McCann, B. E. Scott, B. J. Williamson, J. J. Waggitt, I. Ashton, L. Johanning, P. Blondel, A. Creech, D. Ingram, J. Norris, M. Finn, R. Torres, P. Cazenave, D. Conley, D. Greaves, G. Savidge, E. Armstrong, C. Hall, R. Kennedy \& J. O'Carroll, "Flow and Benthic Ecology 4D - FLOWBEC - An Overview," Proceedings of the $2^{\text {nd }}$ International Conference on Environmental Interactions of Marine Renewable Energy Technologies (EIMR2014), 28 April-02 May 2014, Stornoway, Isle of Lewis, Outer Hebrides, Scotland.

[2] C.M. Senet, J. Seeman and F. Ziemer, "The near-surface current velocity determined from image sequences of the sea surface," IEEE Transactions on Geoscience and Remote Sensing, vol. 39(3), pp 492505, 2001, doi: 10.1109/36.911108

[3] K. Hessner and P.S. Bell, "High resolution current and bathymetry determined by nautical X-band radar in shallow waters," in Proceedings of OCEANS '09, Bremen, Germany, 11-14 $4^{\text {th }}$ May 2009, doi: 10.1109/OCEANSE.2009.5278333

[4] P.S. Bell, "Submerged dunes and breakwater embayments mapped using wave inversions of shore-mounted X-band marine radar data," in Proceedings of the IEEE International Geoscience and Remote Sensing Symposium (IGARSS), pp 4334-4337, 2010, doi: 10.1109/IGARSS.2010.5652634

[5] P.S. Bell, J. Lawrence and J. Norris, "Determining currents from marine radar data in an extreme current environment at a tidal denergy test site," in Proceedings of the IEEE International Geoscience and Remote Sensing Symposium (IGARSS), Munich, Germany, July 2012, doi: 10.1109/IGARSS.2012.6351856

[6] D.L. Codiga, "Unified tidal analysis and prediction using the UTide Matlab functions,” unpublished technical report 2011-01, Graduate Scool of Oceanography, University of Rhode Island, Narragansett, R.I. U.S.A. 